\title{
Genomic analysis of a reef-building coral, Acropora digitifera, reveals complex population structure and a migration network in the Nansei Islands, Japan
}

\author{
Kojin Tsuchiya ${ }^{1}$, Yuna Zayasu ${ }^{2}$, Yuichi Nakajima ${ }^{2}$, Nana Arakaki², Go Suzuki ${ }^{3}$, Nori \\ Satoh $^{2}$, and Chuya Shinzato ${ }^{1}$ \\ ${ }^{1}$ The University of Tokyo Atmosphere and Ocean Research Institute \\ ${ }^{2}$ Okinawa Institute of Science and Technology Graduate University \\ ${ }^{3}$ Japan Fisheries Research and Education Agency Marine Fisheries Research and \\ Development Center
}

December 12, 2021

\begin{abstract}
Understanding the structure and connectivity of coral populations is fundamental for developing marine conservation policies, especially in patchy environments such as archipelagos. The Nansei Islands, extending more than 1,000 km in southwestern Japan, are characterized by high levels of biodiversity and endemism, supported by coral reefs, although precise, detailed genetic attributes of corals are still largely unknown. In this study, we conducted population genomic analyses based on genomewide, single-nucleotide polymorphisms (SNPs) of Acropora digitifera, a common species in the Nansei Islands, for which a complete genome is available. With $\sim 24 \mathrm{x}$ sequencing coverage of entire genomes of 303 colonies collected at 21 locations, we identified more than four million genome-wide SNPs. While population structure analyses suggested weak genetic differentiation among sampled locations, the most southwestern location (the west end of the Yaeyama Islands) was genetically similar to the northernmost location (the Tanegashima Islands), separated by $>1,000 \mathrm{~km}$. Although examination of a migration network found a general tendency of northward migration along the Kuroshio Current, a substantial amount of southward migration was also detected, indicating important contributions of minor ocean currents to coral larval dispersal. Moreover, heterogeneity in the transition of effective population sizes among locations suggests different histories for individual subpopulations. The unexpected complexity of both past and present population dynamics in the Nansei Islands implies that heterogeneity of ocean currents and local environments, past and present, have influenced the population structure of this species, highlighting the importance of local scale assessments for effective coral restoration and management.
\end{abstract}

\section{Hosted file}

Main manuscript.doc available at https://authorea.com/users/450824/articles/549090-genomicanalysis-of-a-reef-building-coral-acropora-digitifera-reveals-complex-populationstructure-and-a-migration-network-in-the-nansei-islands-japan 

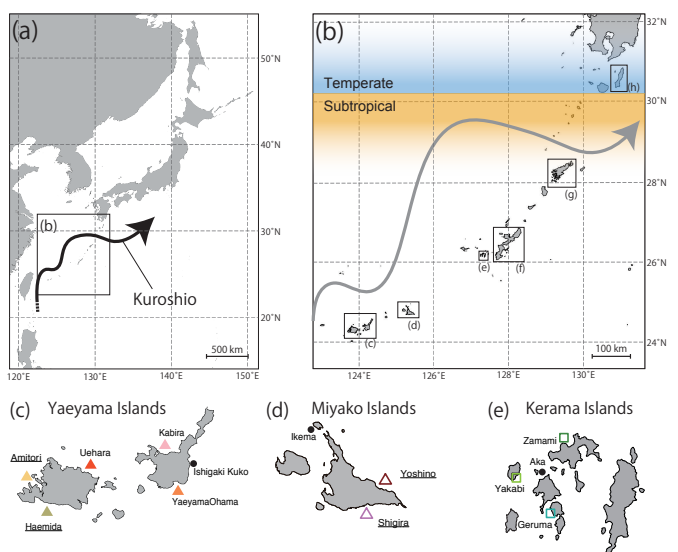

(d) Miyako Islands

(e) Kerama Islands
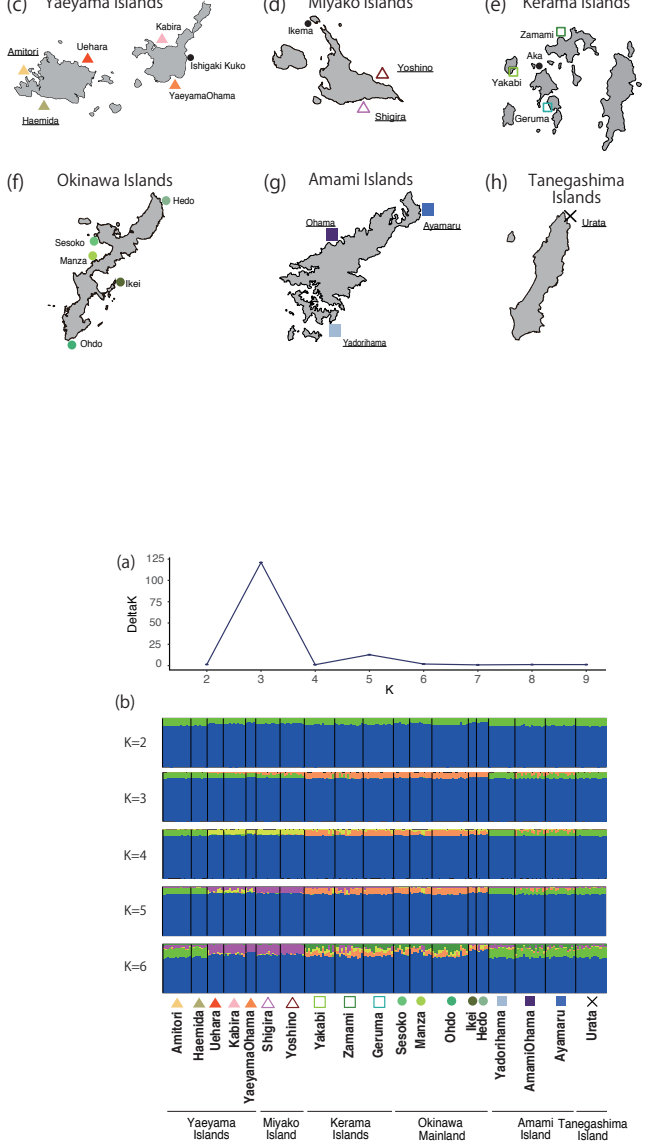


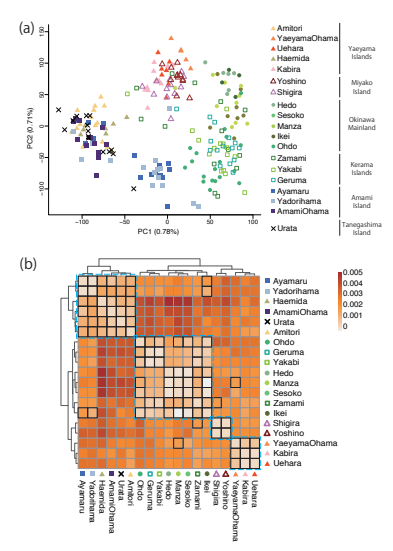

(a)

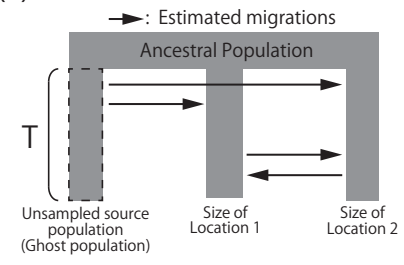

(c)

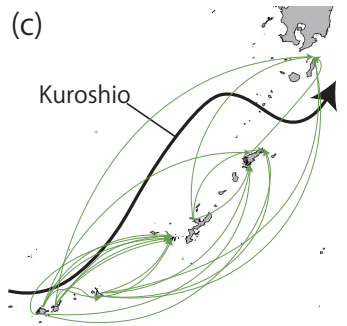

(b)

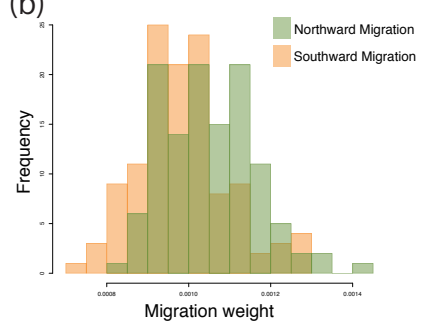

(d)

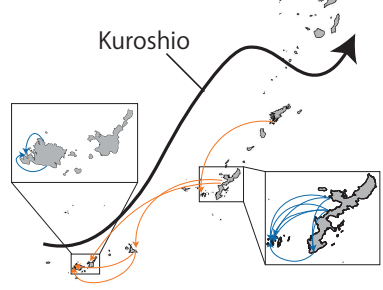




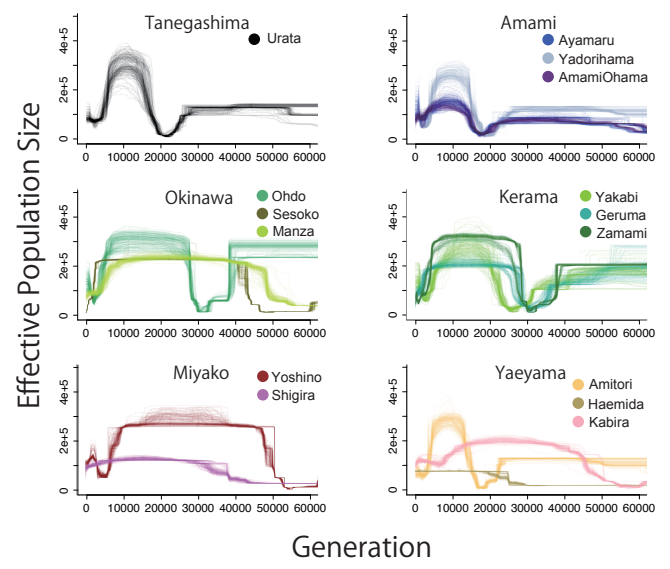

